\title{
ORIGINAL
}

\section{SITUACIÓN ACTUAL EN ESPAÑA DE LOS AEROSOLES INSECTICIDAS REGISTRADOS EN SANIDAD AMBIENTAL PARA USO DOMÉSTICO}

\author{
Josefa Moreno Marí, Amparo Meliá Llácer, María Teresa Oltra Moscardó y Ricardo Jiménez \\ Peydró
}

Laboratorio de Entomología y Control de Plagas. Instituto Cavanilles de Biodiversidad y Biología Evolutiva. Universidad de Valencia. Apartado Oficial 2085. 46071 Valencia.

\section{RESUMEN}

Fundamentos: La reciente transposición de la Directiva de Biocidas a través del Real Decreto 1.054/2002 conllevará un cambio sustancial en distintos aspectos de la Sanidad Ambiental. Para evaluar la incidencia de estos cambios se presenta un análisis de la situación actual, a partir del cual se podrán valorar adecuadamente las consecuencias de la implantación de la Directiva en España, así como establecer los aspectos básicos que se deben analizar con vistas al establecimiento de un Registro de Biocidas acorde con dicho Real Decreto.

Métodos: El análisis se ha efectuado a partir de los datos que figuran en la base de datos del Ministerio de Sanidad y Consumo sobre los insecticidas registrados para Uso Doméstico presentados como aerosol. La elección de los aerosoles se ha realizado por tratarse del tipo de formulación más numerosa y utilizada para uso doméstico.

Resultados: En la formulación de estos aerosoles intervienen 25 insecticidas, 1 desinfectante y 2 sinergizantes. La mayoría de los 298 aerosoles insecticidas presentan algún piretroide en su composición, sólo o en combinación con algún otro compuesto insecticida, desinfectante y/o sinergizante. La mayoría de estos biocidas son mezcla de sustancias activas.

Conclusiones: Del análisis realizado se deduce la necesidad de definir nuevos procedimientos de evaluación de la eficacia de los formulados preparados para el uso (diseño de protocolos de ensayo estandarizados, evaluación de la eficacia sobre las distintas especies plaga, efecto de la mezcla de materias activas, plazos de seguridad,...), además de los aspectos referentes a la toxicología, ecotoxicología o características físico-químicas.

Palabras clave: Pesticidas. Insecticidas. Aerosoles. Salud ambiental. Salud Pública.

\section{Correspondencia:}

Josefa Moreno Marí

Laboratorio de Entomología y Control de Plagas

Instituto Cavanilles de Biodiversidad y Biología Evolutiva

Universidad de Valencia

Apartado Oficial 2085

46071 Valencia.

Correo electrónico: josefa.moreno@uv.es

\section{ABSTRACT}

\section{Current Situation in Spain of Aerosol insect Sprays Registered for Household Use by the Environmental Health Authorities}

Background: The recent transposition of the Biocides Directive by means of Royal Decree 1054/2002 involves a substantial change in different Environmental Health-related aspects. To assess the impact of these changes, an analysis of the current situation is provi ded, based on which the consequences of the implementation of this Directive in Spain may be assessed, in addition to setting out the main aspects which must be analyzed with a view to a Biocides Registry being put into place in accordance with the aforementioned Royal Decree.

Methods: The analysis was made based on the data included in the Spanish Ministry of Health and Consumer Affairs related to aerosol insect sprays registered for Household Use. Aerosols were chosen as a result of their being the type most numerous on the market for household use.

Results: The formulation of these aerosols includes 25 insecticides, 1 disinfectant and 2 synergizers. A majority of the 298 aerosol insect sprays include some pyretroid in their composition, by itself or combined with some other insecticide, disinfectant and/or synergizing compound. Most of these biocides are a mixture of active substances.

Conclusions: Based on the analysis made, the need of setting out new procedures for assessing the effectiveness of the formulas prepared for the use in question (design of standardized testing protocols, assessment of effectiveness on the different pest species, the effect of the mixture of active ingredients, safety timeframes, ...), in addition to the aspects related to toxicology, ecotoxicology or physicochemical characteristics.

Key words: Pesticides. Insecticides. Aerosols. Environmental Health. Public Health. 


\section{INTRODUCCIÓN}

La Directiva de Biocidas ${ }^{1}$, por la que se regula el proceso de autorización de productos biocidas a nivel comunitario, ha sido recientemente transpuesta a la legislación española a través del Real Decreto 1054/ 2002, de 11 de octubre de $2002^{2}$. Este Real Decreto regula el proceso de evaluación para el registro, autorización y comercialización de biocidas. En su artículo 2 se define el término biocida como «las sustancias activas y preparados que contengan una o más sustancias activas, presentados en la forma en que son suministrados al usuario, destinados a destruir, contrarrestar, neutralizar, impedir la acción o ejercer un control de otro tipo sobre cualquier organismo nocivo por medios químicos o biológicos». En el Anexo 5 figura una lista exhaustiva de los veintitrés tipos de productos contemplados, en la que los insecticidas se incluyen en el subgrupo o tipo 18 (T18: Insecticidas, acaricidas y productos para controlar otros artrópodos) y se definen como productos empleados para el control de artrópodos (insectos, arácnidos y crustáceos, fundamentalmente).

La Dirección General de Salud Pública (Subdirección General de Sanidad Ambiental) del Ministerio de Sanidad y Consumo es la unidad competente en España para la aplicación de la legislación europea sobre sustancias y preparados químicos peligrosos. Además, la Dirección General de Salud Pública es responsable del Registro de Plaguicidas de Uso en Salud Pública (Biocidas), del Sistema de Notificación de Sustancias Nuevas, de la Evaluación del Riesgo de Sustancias Existentes, de la Directiva de Limitaciones, de la Exportación-Importación de Sustancias y Productos Químicos Peligrosos, del Registro de Sprays de Defensa Personal, de la evaluación de la peligrosidadtoxicidad para los Plaguicidas Fitosanitarios, Zoosanitarios y Fertilizantes, y homologación de productos para el tratamiento de piscinas ${ }^{3}$.
Mientras algunos Estados de la Unión Europea no han tenido ningún tipo de control sobre estos productos, en España desde 1983, se mantiene un registro para la autorización de los plaguicidas no agrícolas ${ }^{3,4}$. La legislación previa existente está constituida por el Real Decreto 3349/19835 y posteriores modificaciones. Dicho decreto tiene como objeto principal la ordenación técnicosanitaria de estos productos en lo que concierne a salud pública, con el fin de prevenir accidentes e intoxicaciones y evitar o limitar los peligros asociados a sus usos. El Real Decreto 162/19916 modificó la Reglamentación Técnico Sanitaria (RTS) con el fin de adaptarla a la Ley General de Sanidad, atribuyendo al Ministerio de Sanidad y Consumo la posibilidad de limitar la comercialización de preparados cuando supongan un peligro para la salud humana o su seguri$\mathrm{dad}^{3}$. El Real Decreto 443/19947 modificó una vez más la RTS, con el fin de incluir a los plaguicidas de uso en la industria alimentaria entre los que deberían inscribirse en el Registro de la Dirección General de Salud Pública. Además, la RTS fue completada mediante diversas órdenes relativas a la inscripción y funcionamiento del Registro de Establecimientos y Servicios Plaguicidas, a la normativa reguladora del Libro Oficial de Movimiento de plaguicidas peligrosos y a la homologación de los cursos de capacitación para realizar tratamientos con plaguicidas ${ }^{3,4}$.

En función de esta legislación, desde 1983 se han venido registrando varios tipos de productos considerados ahora biocidas en la Directiva 98/8/CE como son: plaguicidas de uso doméstico, plaguicidas de uso ambiental, de uso en la industria alimentaria, de uso en higiene personal y desinfectantes, así como los productos zoosanitarios.

Para la inscripción de estos productos, es necesario evaluar sus aspectos de peligrosidad para la salud humana. Para ello se exige a los fabricantes la siguiente información básica sobre los plaguicidas: clasificación toxicológica, pictograma, frases R (consejos 
de prudencia), frases $\mathrm{S}$ (recomendaciones y tratamiento médico en casos de intoxicación o accidente), aplicaciones y usos autorizados, modo de empleo, incluyendo plazo de seguridad o periodo de tiempo que debe transcurrir desde su aplicación hasta la entrada en las áreas o recintos tratados. Sin embargo, hay otros muchos aspectos actualmente contemplados por la Directiva de Biocidas y por el Real Decreto 1054/2002 que deberían incluirse en los requisitos para la inscripción de los biocidas y entre los que destacaremos las evaluaciones de eficacia y ecotoxicológicas. En este sentido, el objetivo del estudio que se presenta es el establecimiento de la situación de partida previa a la completa aplicación de la Directiva de Biocidas, para lo cual resulta de gran interés efectuar un estudio de la situación actual a partir de los datos existentes en la base de datos del Registro Oficial de Plaguicidas de Uso en Salud Pública en España, a partir del cual se podrán valorar adecuadamente las consecuencias de la implantación de la nueva Directiva en España. Este análisis permitirá también definir algunos de los aspectos básicos que se deben analizar a fin de establecer un nuevo Registro de Biocidas acorde con lo establecido en el Real Decreto 1.054/2002.

Dado el elevado número de materias activas y formulados que figuran en el Registro y la gran variedad de los mismos en cuanto a su eficacia, el estudio cuyos resultados se presentan tiene por objeto realizar un análisis exhaustivo de los biocidas con efecto insecticida (Grupo Principal 3; Tipo de producto 18) autorizados para Uso Doméstico y formulados como aerosoles, por constituir este grupo uno de los más importantes en cuanto al número de biocidas inscritos y a su uso por la población en general.

\section{MATERIAL Y MÉTODOS}

El estudio se ha realizado a partir de la información que figura en la base de datos del Registro Oficial de Plaguicidas de Uso en Salud Pública de España para los insecticidas formulados como aerosoles y registrados para uso doméstico con fecha 7 de febrero de $2002^{8}$. Para el análisis de los datos recogidos en dicho Registro se han considerado los siguientes parámetros:

Composición (tipo de materias activas presentes en los formulados, número de materias activas que conforman los formulados, combinación de materias activas en los formulados y adición de sinergizantes y/o desinfectantes), usos autorizados, especies susceptibles y plazos de seguridad.

\section{RESULTADOS}

En el momento de realizar la revisión del Registro Oficial de Plaguicidas de Uso en Salud Pública figuran en el mismo un total de 1.396 insecticidas registrados en Sanidad Ambiental (Grupo Principal 3; Tipo de producto 18 según la Directiva 98/8/CE), de los que el 39\% (543 formulados) están registrados para uso doméstico mientras que los restantes $(61 \%)$ lo están únicamente para uso profesional (uso ambiental y/o en industria alimentaria).

Por lo que se refiere al tipo de formulación, la mayoría de los compuestos registrados para uso doméstico se formulan para su aplicación como líquidos $(81,2 \%$ de $\operatorname{los}$ insecticidas registrados). De todas las posibles formulaciones (concentrados emulsionables, aerosoles, polvos mojables, polvos solubles, microencapsulados, ....), los aerosoles constituyen el grupo más numeroso y más utilizado para uso doméstico ya que, como tal, se formulan un total de 298 compuestos, lo que supone el $54,9 \%$ de los insecticidas registrados para uso doméstico.

En la formulación de estos aerosoles intervienen 25 materias o sustancias activas (16 piretroides/piretrinas, 3 organofosforados; 3 carbamatos, 2 reguladores del creci- 
miento de insectos (RCI) y 1 sal), 1 desinfectante y 2 sinergizantes (tabla 1 ).

La mayoría de los 298 aerosoles insecticidas presentan algún piretroide en su composición (294 formulados), bien sólo (253 formulados), bien en combinación con algún otro compuesto insecticida, desinfectante y/o sinergizante (41 formulados) (tabla 2). Únicamente 4 formulados no presentan piretroides y/o piretrinas, de los que 1 sólo presenta organofosforados, 1 únicamente carbamatos, 1 es combinación de carbamatos y organofosforados y 1 combina carbamatos y RCI.

Una revisión detallada de su composición muestra que la mayoría de estos biocidas son mezcla de sustancias activas: 15 formulados presentan una sola sustancia activa, mientras que los 283 restantes son mezclas de sinergizantes e insecticidas (de una o varias familias) o de insecticidas (de una o varias familias), sinergizantes y desinfectantes. La gran mayoría de los formulados presentan 2 (191 formulados) ó 3 (77 formulados) materias activas en su composición, si bien en algunos casos llegan a presentar hasta 4 (11 formulados) e incluso 5 (4 formulados).

Las materias activas más habituales han resultado ser la tetrametrina, presente en 250 formulados, y el fenotrín, materia activa que interviene en 132 formulados. Por lo que se refiere a la combinación de biocidas, la más habitual ha sido tetrametrina y fenotrín (99 formulados), seguida de tetrametrina y cifenotrín (14 formulados) y de la combinación

Tabla 1

Relación de materias activas, número CAS (Chemical Abstract Service) y EINECS (European Inventory of Existing Commercial Chemical Substances)

\begin{tabular}{|c|c|c|c|c|}
\hline Tipos de Biocida & Tipo de Insecticida & Material activa & $\mathbf{N}^{\mathrm{a}} \mathrm{CAS}$ & $\mathbf{N}^{\circ}$ EINECS \\
\hline \multirow[t]{26}{*}{ Insecticidas (25) } & \multirow[t]{17}{*}{ Piretroides/ piretrinas (16) } & Praletrina & $23031-36-9$ & $245-387-9$ \\
\hline & & Cifenotrín & $39515-40-7$ & $254-484-5$ \\
\hline & & Tetrametrina & $7696-12-0$ & $231-711-6$ \\
\hline & & Aletrina & $584-79-2$ & $209-542-4$ \\
\hline & & S-bioaletrina & $28434-00-6$ & $249-013-5$ \\
\hline & & Fenotrín & $26046-85-5$ & $247-431-2$ \\
\hline & & Permetrina & $52645-53-1$ & $258-067-9$ \\
\hline & & Ciflutrín & $68359-37-5$ & $269-855-7$ \\
\hline & & Transflutrín & $118712-89-3$ & 0835 \\
\hline & & Bioresmetrina & 28434-01-7 & $249-014-0$ \\
\hline & & Alfa-cipermetrina & $67375-30-8$ & $257-842-9$ \\
\hline & & Cipermetrina & $52315-07-8$ & $257-842-9$ \\
\hline & & Empentrín & $54406-48-3$ & $259-154-4$ \\
\hline & & Deltametrina & $52918-63-5$ & $258-256-6$ \\
\hline & & Imiprotrín & $72963-72-5$ & 3614 \\
\hline & & Piretrina I & $121-21-1$ & $204-455-8$ \\
\hline & & Piretrina II & $121-29-9$ & $204-462-6$ \\
\hline & \multirow[t]{3}{*}{ Organofosforados (3) } & Fenitrotión & $122-14-5$ & $204-524-2$ \\
\hline & & Clorpirifos & $2921-88-2$ & $220-864-4$ \\
\hline & & Diazinón & $333-41-5$ & $206-373-8$ \\
\hline & \multirow[t]{3}{*}{ Carbamatos (3) } & Propoxur & 114-26-1 & $204-043-8$ \\
\hline & & Metomilo & $16752-77-5$ & $240-815-0$ \\
\hline & & Bendiocarb & $22781-23-3$ & $245-216-8$ \\
\hline & \multirow[t]{2}{*}{ Reguladores crecimiento de insectos (2) } & Piriproxifen & $95737-68-1$ & 0818 \\
\hline & & Diflubenzurón & $35367-38-5$ & $252-529-3$ \\
\hline & Sales (1) & Benzoato de bencilo & $120-51-4$ & $204-402-9$ \\
\hline \multirow[t]{2}{*}{ Sinergizantes (2) } & & Butóxido de piperonilo & $51-03-6$ & $200-076-7$ \\
\hline & & $\begin{array}{l}\text { MGK-264 (N-octil- } \\
\text { bicicloheptanodicarboximida) }\end{array}$ & $113-48-4$ & $204-029-1$ \\
\hline Sesinfectantes (2) & & Trietilenglicol & $112-27-6$ & $203-953-2$ \\
\hline
\end{tabular}


Tabla 2

Tipos de materias activas presentes en los formulados

\begin{tabular}{|c|c|}
\hline \multicolumn{1}{|c|}{ Materias activas } & No $^{0}$ Biocidas \\
\hline Con piretroides & 294 \\
\hline Sólo piretroides & 253 \\
\hline Piretroides con organofosforados & 17 \\
\hline Piretroides con carbamatos & 16 \\
\hline Piretroides con organofosforados y carbamatos & 4 \\
\hline Piretroides con reguladores del crecimiento & 1 \\
\hline Piretroides con sales & 2 \\
\hline Piretroides con desinfectantes & 4 \\
\hline Sin piretroides & 1 \\
\hline Sólo carbamatos & 1 \\
\hline Organofosforados con carbamatos & 1 \\
\hline Carbamatos con reguladores del crecimiento & 1 \\
\hline
\end{tabular}

tetrametrina, permetrina y butóxido de piperonilo (13 formulados).

Por lo que se refiere a la adición de sinergizantes y/o desinfectantes, de los 298 formulados estudiados, 104 presentan algún sinergizante en su composición y sólo 2 combinan insecticidas con desinfectantes (tabla 2). Todos los formulados con sinergizante presentan algún piretroide en su composición. De los 2 sinergizantes registrados (butóxido de piperonilo y N-octil-bicicloheptanodicarboximida (MGK-264)), la gran mayoría presentan butóxido de piperonilo (101 formulados).

En ninguno de los aerosoles registrados para uso doméstico se precisan las especies susceptibles a su acción. Debido a esto, no existe ninguna limitación en su empleo si exceptuamos la puntualización para algunos respecto a que su empleo sobre plantas se encuentra condicionado a su inscripción en el Registro Oficial de Productos Fitosanitarios.

En España no se exige el establecimiento de plazo de seguridad para los aerosoles registrados para uso doméstico, cosa por otro lado lógica, puesto que se trata de compuestos que se pueden aplicar en presencia de personas o animales. Sin embargo, es relevante que para algunos de estos formulados sí se define un plazo de seguridad en su uso profesional en Sanidad Ambiental o en la Industria Alimentaria y, en particular, resulta llamativo el hecho de que para algunos el plazo de seguridad sea superior al mínimo establecido por la legislación española, que en este momento se sitúa en 12 horas desde la aplicación.

\section{DISCUSIÓN}

El análisis de la situación existente en España antes de la transposición de la Directiva 98/8/EC pone en evidencia algunas cuestiones que merecen ser tenidas en cuenta ante la próxima adaptación del sistema español a la normativa europea y que pueden resultar de interés para otros países miembros de la Comunidad Europea.

La revisión del Registro Oficial de Plaguicidas de Uso en Salud Pública español ha 
revelado que los aerosoles constituyen el grupo más numeroso y más utilizado para uso doméstico ya que como tal se formulan el 54,9\% de los insecticidas registrados para dicho uso.

Una primera cuestión que llama poderosamente la atención es la ausencia de datos sobre la eficacia de los aerosoles insecticidas registrados para uso doméstico. Todos ellos se registran de forma genérica sin indicación de las especies realmente susceptibles, cuando del análisis de su composición se pueden deducir diferencias importantes en cuanto a la eficacia según la especie plaga considerada. Sólo en algunos casos se proporciona una ligera indicación de las especies para las cuales se recomiendan de forma preferente, precisión que generalmente se señala en el nombre del producto pero no en la etiqueta. Vistas las especificaciones establecidas en la Directiva 98/8/EC, así como las recomendaciones del Technical Guidance Document del European Chemical Bureau ${ }^{9}$, del Environment Directorate of the Organisation for Economic Co-operation and Development ${ }^{10,11}$, del European Chemical Industry Council $^{12}$ y del Environmental Protection Agency of United States ${ }^{13,14}$, la nueva normativa deberá contemplar necesariamente una evaluación de la eficacia de estos compuestos sobre las distintas especies plaga a través de métodos estandarizados.

La mayoría de estos formulados presentan algún piretroide en la composición pero existe una gran variedad en cuanto a las materias activas que los componen, de lo que surge una cuestión en cuanto a si es necesario ser más selectivos en cuanto a las materias activas autorizadas para uso doméstico partiendo para ello de los estudios toxicológicos, ecotoxicológicos y de eficacia.

De los 298 formulados estudiados 266 son mezclas de varios biocidas. En algunos casos están formados incluso por 5 biocidas. De ello se deduce que debemos plantearnos si es realmente necesario recurrir al empleo de mezclas para el control de las plagas domésticas y si éstas mejoran realmente la eficacia sin incrementar los riesgos. Teniendo en cuenta además la estrecha relación entre mezcla y aparición de resistencias, este aspecto debería ser prioritario en la evaluación de los formulados para su autorización. En este sentido consideramos altamente positiva la inclusión en la Directiva 98/8/EC de la exigencia de incluir no sólo la evaluación de las materias activas sino también de los formulados preparados para su empleo, así como la inclusión en dicha evaluación de los estudios sobre resistencia. Todo ello contribuirá sin duda a lograr uno de los objetivos finales de dicha Directiva: la reducción de los riesgos asociados al empleo de estos biocidas.

En relación con la conveniencia o no de las mezclas y atendiendo al tipo de combinación en función del tipo de materias activas, el estudio realizado ha puesto en evidencia que 45 formulados de los 298 registrados son combinación de más de una materia activa de distinta familia. A partir de estos datos se plantea otra cuestión: ¿la mezcla de materias activas de distinta familia contribuye a una mejor eficacia del formulado sin incremento de los riesgos? Entre las distintas combinaciones destacan las siguientes: piretroides con piretroides, piretroides con organofosforados, piretroides con carbamatos, piretroides con organofosforados y carbamatos, carbamatos con organofosforados, piretroides con R.C.I., o carbamatos con R.C.I.. Algunas de estas combinaciones resultan sorprendentes como es el caso, por ejemplo, de la combinación de piretroides y RCI ya que no se trata de una combinación lógica desde un punto de vista biológico. Dadas las características de los ambientes en los que se aplican estos aerosoles y el tipo de aplicación que de los mismos se realiza, los datos disponibles actualmente sobre estas materias activas no apoyan un incremento de eficacia. Tampoco parece lógico ni necesario formular con más de un piretroide ya que 
generalmente esta combinación no se traduce en un incremento de la eficacia y existe un incremento de los riesgos, puesto que no se conoce realmente cuál es realmente la consecuencia de estas mezclas en la mayoría de los casos (¿sinergismo?, ¿potenciación?, ¿se trata de mezclas que potencian las materias activas sin modificar los riesgos?, ¿los incrementan?). Debemos tener en cuenta además que existe una clara relación entre las mezclas y la aparición de resistencias y que no todas las materias activas o combinación de las mismas son igual de eficaces contra las distintas especies plaga. A la vista de lo indicado, y teniendo en cuenta que por tratarse de insecticidas para uso doméstico es el usuario el que finalmente decide el uso concreto que del mismo va a realizar, parece recomendable establecer un nuevo sistema de etiquetado en el que se incluya entre otros datos una mayor información sobre las especies y situaciones en las que se recomienda su empleo, restringiendo o limitando el empleo de mezclas en lo posible. De todo ello se deduce la imperiosa necesidad de modificar los criterios para la evaluación de los biocidas, ampliando el ámbito de aplicación de los mismos a los formulados tal y como son utilizados por el usuario.

Otra de las cuestiones que llama poderosamente la atención es la adición de sinergizantes y la concentración de los mismos en los formulados. Atendiendo a los nuevos criterios establecidos en la Directiva 98/8/EC y en vistas a conseguir un mejor equilibrio en el balance riesgo-beneficio, tanto los efectos de la adición de estos sinergizantes como el efecto concentración, deben ser convenientemente evaluados.

De los resultados del estudio realizado por el Environment Directorate of the Organisation for Economic Co-operation and Development ${ }^{10}$ y comparando los mismos con la situación en España, resulta evidente que se hace absolutamente necesario proceder a una homogenización de los criterios y categorías para la clasificación de los biocidas en las distintas categorías toxicológicas, físicoquímico-técnicas, estableciendo los mismos criterios y los mismos valores. Se recomienda también exigir una mayor precisión en cuanto a la información que sobre su clasificación se indican en la base de datos del Registro Oficial de Plaguicidas, así como en las etiquetas. En relación con los usos autorizados debería establecerse una clasificación más específica adaptada a lo establecido en la Directiva 98/8/EC, similar a la establecida recientemente por Baumann y colaboradores ${ }^{15}$, con una clara separación entre los formulados para uso profesional y los de uso no-profesional, así como en función de la situación en la que se vaya a proceder a su empleo.

Otro de los aspectos llamativos es el establecimiento de plazos de seguridad para algunos formulados, registrados tanto para uso profesional como para uso no-profesional, cuando su empleo sea en el ámbito profesional. La legislación española establece distintos plazos de seguridad (0 horas, 12 horas, 24 horas, 48 horas) para los plaguicidas registrados para Uso en Sanidad Ambiental y/o en la Industria Alimentaria, no estableciéndose dicho plazo para los plaguicidas registrados para Uso Doméstico. En este sentido, y para un mismo formulado, podemos encontrarnos con que un determinado producto esté registrado tanto para Uso Doméstico, y por tanto sin plazo de seguridad, como para Uso Ambiental y/o en la Industria Alimentaria, en este caso con un plazo de seguridad de 12 ó 24 horas, sin que varíen cuestiones como la concentración o el tipo de aplicación. Si tenemos en cuenta que el plazo de seguridad es el periodo de tiempo necesario para lograr el efecto biocida y se define como el intervalo de tiempo que debe de transcurrir entre dos aplicaciones, entre la aplicación y el uso de los productos tratados o entre la aplicación y la entrada de personas o animales en las áreas tratadas con un biocida, incluyéndose en dicho intervalo el tiempo necesario para la descontaminación y la adecuada ventilación de las áreas tratadas, y 
considerando que es muy difícil que el usuario doméstico cumpla con los plazos de seguridad, surge una cuestión crucial referente a si no se debería proporcionar al usuario no-profesional indicaciones sobre si la aplicación debe realizarse en ausencia de personas y animales, el periodo de tiempo que debe transcurrir entre la aplicación y la entrada de personas o animales o el tiempo que debe pasar entre tratamientos. De no hacerlo así se presupone que no existen riesgos para el usuario y, en este caso, ¿cuáles son las razones que llevan a la exigencia del cumplimiento de plazos de seguridad para los profesionales? Por supuesto, existen aspectos como el tamaño de la población que podría verse afectada, o la necesidad de establecer de forma clara que no se puede proceder a una nueva aplicación hasta que no transcurra dicho plazo a fin de evitar mezclas espaciales, que justifican plenamente la adopción de plazos de seguridad en su empleo profesional, pero consideramos necesaria la inclusión de recomendaciones en este sentido para el usuario no-profesional.

De lo indicado para los aerosoles insecticidas registrados para Uso Doméstico, y que puede resultar en gran medida extrapolable a otros biocidas, se deduce la necesidad de proceder a la implantación con la mayor brevedad posible de la Directiva 98/8/EC, en particular en lo relativo a la evaluación de la eficacia de los formulados (diseño de protocolos de ensayo estandarizados, evaluación de la eficacia sobre las distintas especies plaga, valoración del efecto de la mezcla de materias activas y/o adición de sinergizantes, estimar el efecto concentración, establecer plazos de seguridad, usos, condiciones de aplicación, etiquetado,...), además de los aspectos referentes a la toxicología, ecotoxicología o características físico-químicas. El análisis de la situación actual nos permitirá en un futuro valorar la mejora que la implementación de la Directiva 98/8/EC puede conllevar en la optimización del empleo de biocidas.

\section{BIBLIOGRAFÍA}

1. Diario Oficial de las Comunidades Europeas. Directiva 98/8/CE relativa a la comercialización de biocidas. DOCE núm L 123, 24/4/1998.

2. Boletín Oficial del Estado. Real Decreto $1054 / 2002$ por el que se regula el proceso de evaluación para el registro, autorización y comercialización de biocidas. BOE núm, 247, 15/10/2002.

3. Vargas F. Salud Pública y Productos Químicos en España y Europa: ¿hacia dónde vamos? 7. ${ }^{\text {a Jornada }}$ Técnica SESA. Seguridad Química. Ministerio de Sanidad y Consumo; 2000. p. 81.

4. Peña E. y Gómez E., editores. Evaluación Toxicológica de los plaguicidas y la Sanidad Ambiental. Monografía SESA/AET. Murcia, 2000. p. 81.

5. Boletín Oficial del Estado. Real Decreto $3349 / 1983$ de 30 de noviembre, por el que se aprueba la Reglamentación Técnico Sanitaria para la fabricación, comercialización y utilización de plaguicidas. BOE núm 19, 24/1/1984.

6. Boletín Oficial del Estado. Real Decreto 162/1991 por el que se modifica la Reglamentación TécnicoSanitaria para la fabricación, comercialización y utilización de los plaguicidas. BOE núm 41, $15 / 2 / 1991$.

7. Boletín Oficial del Estado. Real Decreto 443/1994 por el que se modifica la Reglamentación TécnicoSanitaria para la fabricación, comercialización y utilización de los plaguicidas. BOE núm. 76, 30/3/1994.

8. Ministerio de Sanidad y Consumo. Registro Oficial de Plaguicidas de Uso en Salud Pública (7 de febrero de 2002). Disponible en: www.msc.es/salud/ambiental/home.htm.

9. European Chemical Bureau. Technical guidance document in support of the Directive 98/8/EC concerning the placing of biocidal products on the market: Guidance on data requeriments for active substances and biocidal products. Bruselas: European Chemical Bureau; 2000. p.143.

10. OCDE. Report of the Survey of OECD Member Countries' Approaches to the Regulation of Biocides. OECD Environmental Health and Safety $\mathrm{Pu}-$ blications. Series on Pesticides No. 9. Organisation for Economic Co-operation and Development; 1999. p. 545.

Rev Esp Salud Pública 2003, Vol. 77, N. ${ }^{\circ} 3$ 
11. OECD. Overview of efficacy testing methods for biocides. Environmental Health and Safety Publications. Organisation for Economic Co-operation and Development; 2000. p.98.

12. CEFIC. Comments and Proposals concerning efficacy data requeriments in the context of EU Biocides Directive (98/8/EC). CEFIC; 1996.
13. US-EPA. Reporting Requirements for risk/benefit information. Fed Regist 1997; 62(182): 49370-95.

14. US-EPA. USEPA Guidelines OPPTS 810; 2002.

15. Baumann W, Hesse K, Pollkläsner D, Kummerer K, Kumpel T. 2000. Gathering and Review of Environmental Emisión Scenarios for Biocides. Bruselas: European Chemical Bureau. Institute for Environmental Research; 2000. p.236. 